\title{
Technological improvements for whole cell cryo-ET of respiratory syncytial virus infected cells
}

\author{
Bryan Sibert ${ }^{1}$, Joseph $\mathrm{Kim}^{2}$, Jae Yang ${ }^{1}$ and Elizabeth Wright ${ }^{1}$ \\ ${ }^{1}$ Department of Biochemistry, University of Wisconsin-Madison, Madison, Wisconsin, United States, \\ ${ }^{2}$ Department of Chemistry, University of Wisconsin-Madison, Madison, Wisconsin, United States
}

Respiratory syncytial virus (RSV) is a common respiratory virus that causes over 235,000 hospitalizations in the US each year $[1,2]$. There are currently no approved treatments or vaccines. RSV virions are enveloped filamentous virions that are highly pleomorphic, but generally range in length from 1-4 $\mu \mathrm{m}$ with a diameter of $\sim 130 \mathrm{~nm}$ [3]. The RSV matrix protein (M) has been crystallized as both a monomer and a dimer and experiments have shown that dimerization is required to assemble filamentous virions [4, 5]. However, the organization of matrix within the virion has not been determined. The structure and organization matrix proteins or matrix protein equivalents of other paramyxoviruses has been solved by cryo-EM, cryo-ET, and sub-tomogram averaging [6-8].

Previously, we reported our observation of a lattice-like density in the M-protein layer from cryo-ET of RSV virions. Using sub-tomogram averaging, we were able to further resolve the structure of RSV M (Fig. 1). The lattice is helical-like in nature, with a helical angle of $\sim 45^{\circ}$ relative to the long axis of the virus. The densities in the lattice have a center-center spacing of approximately $5.2 \mathrm{~nm}$ and $6.4 \mathrm{~nm}$. This spacing is consistent with each subunit containing an $\mathbf{M}$ dimer. The average presented here includes particles from a range of virion diameters and local membrane curvature. Work is ongoing to determine the effect of local curvature on lattice spacing.

Using whole-cell cryo-ET to study RSV or other viruses requires a sufficiently low cell density to provide open areas on the foil for imaging of the cell periphery or released virions and to prevent the formation of overly thick ice while plunge freezing. The cell distribution across the grid is often uneven with many cells on grid bars or clustered together making them unsuitable for tomography. Additionally, only a subset of cells are infected and actively producing virus, further reducing the number of potential targets per grid. For our RSV studies we use a recombinant RSV strain that expresses a fluorescent protein in actively replicating cells. Correlative light- and electron- microscopy (CLEM) is useful for rapidly identifying and locating potential regions of interest on the grid during cryo-EM [9]. However, this does not increase the number of potential targets per-grid.

To further optimize our whole-cell tomography workflows, we began micropatterning TEM grids to direct cell growth and positioning on the grids [10-12]. Briefly, an anti-fouling layer is deposited on the grid and subsequently ablated in targeted areas using a UV-laser based micropatterning system. An extra-cellular matrix (ECM) protein is then deposited onto the grid that will adhere only to the patterned areas. During cell seeding the cells preferentially adhere to and grow along the arears of ECM. This results in more even cell distribution across the grid and allows for the positioning of cells within certain areas, such as the center of the grid square. BEAS-2B human airway epithelial cells. (Fig. 2), HeLa cells, and other cell types grown on patterned grids are susceptible to RSV infection and suitable for cryo-ET. Future work will focus on extending the technological capabilities of micropatterning for a range of cryo-ET studies of RSV-infected cells, with the patterning making a direct contribution. For example, patterning will support coordinated cryo-CLEM studies that include cryo-FLM, cryo-FIB-SEM, and cryo-ET data collection and analysis [13]. 

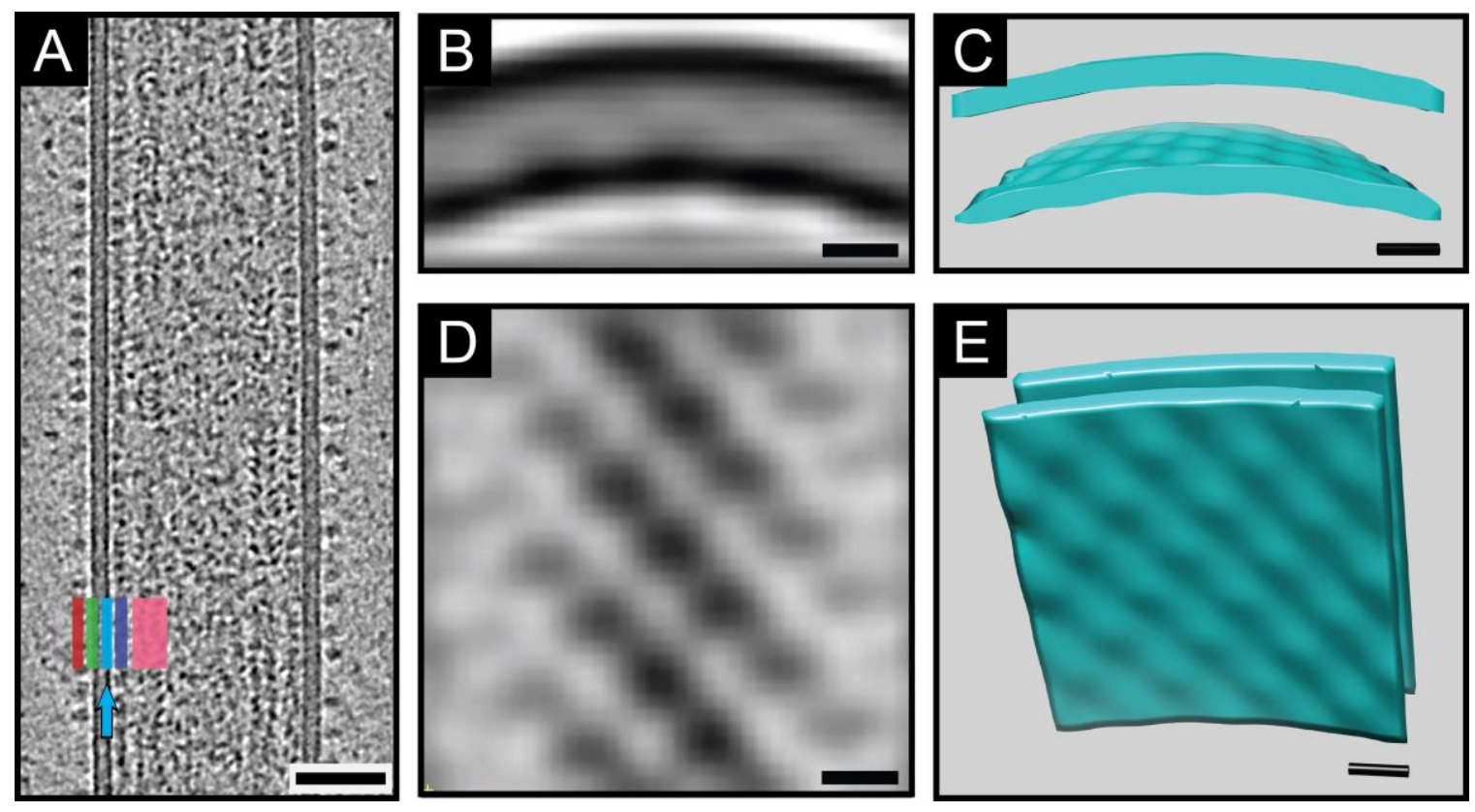

Figure 1. Subtomogram averaging of RSV matrix (M). Micropatterned TEM grid with RSV infected BEAS-2B cells. A) Fluorescently conjugated fibrinogen on a patterned TEM grid (bleed-through from cells in B is visible in some areas). B) Fluorescent image of cells grown on a micropatterned grid and expressing mKate 2 from infection with RSV A2-mK+. C) Low mag TEM map of grid in A and B. A,B,C are roughly correlated in scale and position; the center of each grid is marked with a star. Scale bars are $500 \mu \mathrm{m}$. Fluorescent images are pseudocolored. D) TEM montage of infected cell on patterned grid square. Scale bar is $5 \mu \mathrm{m}$. E) Projection of slices (7nm thick) from tomogram of RSV taken on micropatterned grid. Scale bar is $100 \mathrm{~nm}$. A) Projection of slices (7nm thick) from tomogram of RSV virion. Densities from multiple components are highlighted with colors near the bottom left: red - F; green - membrane; cyan - M; purple - M2-1; pink - nucleocapsid. Matrix is also indicated with an arrow. B) X,Z slice of the electron density map from subtomogram averaging of RSV M. Viral membrane is on top and matrix is the layer underneath. C) Isosurface projection from map in B. D) X,Y slice from the matrix layer of the map in B. E) Isosurface projection from map in B with the matrix layer facing the viewer. B,C,D,E have been lowpass filtered to $30 \AA$ A. Scale bar in A is $50 \mathrm{~nm}$; scale bar in B,C,D,E is $5 \mathrm{~nm}$. 

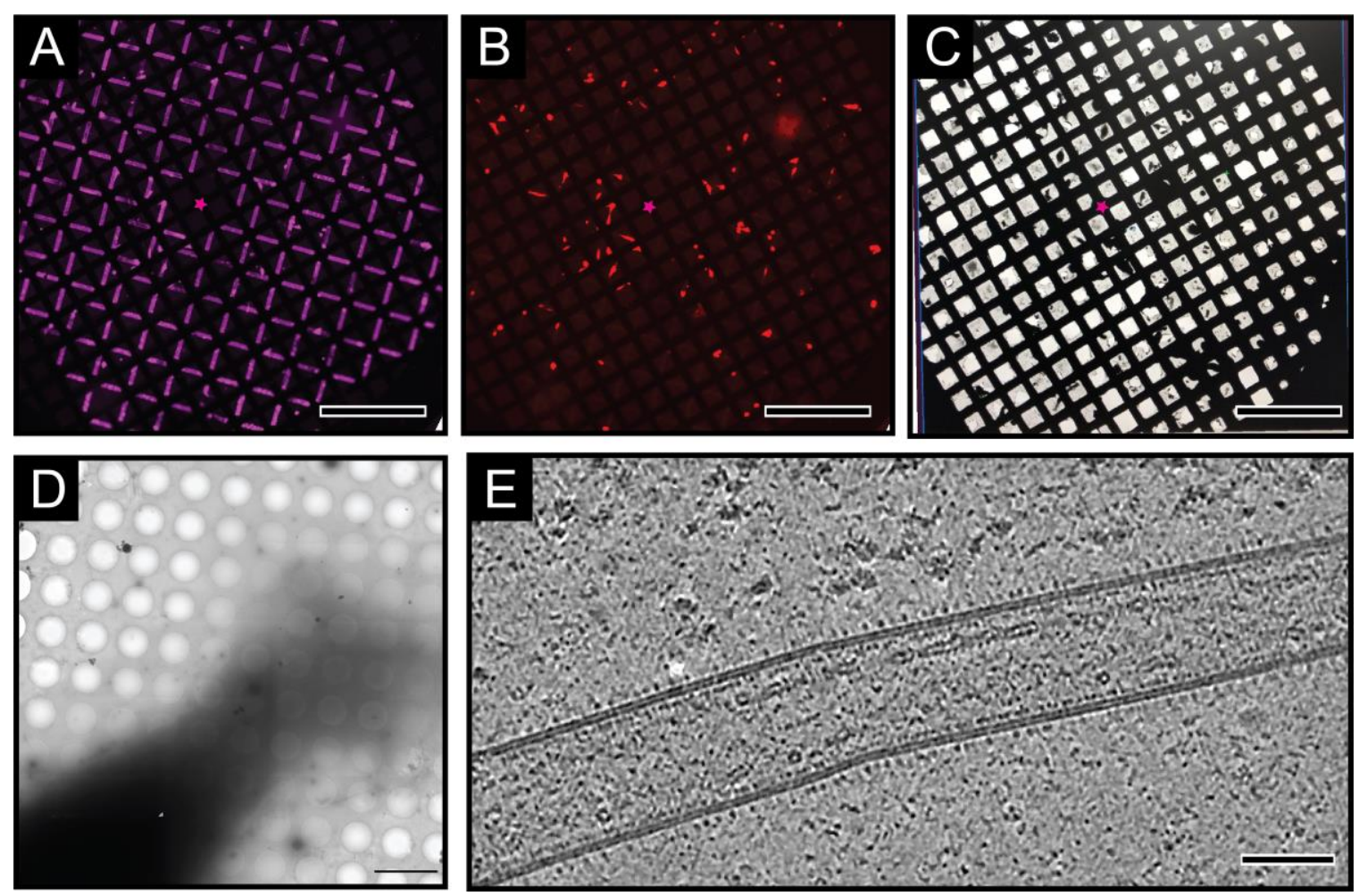

Figure 2. Micropatterned TEM grid with RSV infected BEAS-2B cells. A) Fluorescently conjugated fibrinogen on a patterned TEM grid (bleed-through from cells in B is visible in some areas). B) Fluorescent image of cells grown on a micropatterned grid and expressing mKate 2 from infection with RSV A2-mK+. C) Low mag TEM map of grid in A and B. A,B,C are roughly correlated in scale and position; the center of each grid is marked with a star. Scale bars are $500 \mu \mathrm{m}$. Fluorescent images are pseudocolored. D) TEM montage of infected cell on patterned grid square. Scale bar is $5 \mu \mathrm{m}$. E) Projection of slices ( $7 \mathrm{~nm}$ thick) from tomogram of RSV taken on micropatterned grid. Scale bar is $100 \mathrm{~nm}$.

References

1. Rha, B., et al., Respiratory Syncytial Virus-Associated Hospitalizations Among Young Children: 2015 2016. Pediatrics, 2020.

2. Falsey, A.R., et al., Respiratory syncytial virus infection in elderly and high-risk adults. N Engl J Med, 2005.

3. Ke, Z., et al., The Morphology and Assembly of Respiratory Syncytial Virus Revealed by Cryo-Electron Tomography. Viruses, 2018.

4. Money, V.A., et al., Surface features of a Mononegavirales matrix protein indicate sites of membrane interaction. Proc Natl Acad Sci U S A, 2009.

5. Forster, A., et al., Dimerization of matrix protein is required for budding of respiratory syncytial virus. J Virol, 2015.

6. Wan, W., et al., Ebola and Marburg virus matrix layers are locally ordered assemblies of VP40 dimers. Elife, 2020.

7. Battisti, A.J., et al., Structure and assembly of a paramyxovirus matrix protein. Proc Natl Acad Sci U S A, 2012.

8. Ke, Z., et al., Promotion of virus assembly and organization by the measles virus matrix protein. Nat Commun, 2018. 
9. Yang, J.E., et al., CorRelator: Interactive software for real-time high precision cryo-correlative light and electron microscopy. J Struct Biol, 2021.

10. Toro-Nahuelpan, M., et al., Tailoring cryo-electron microscopy grids by photo-micropatterning for in-cell structural studies. Nature Methods, 2020.

11. Engel, L., et al., Extracellular matrix micropatterning technology for whole cell cryogenic electron microscopy studies. J Micromech Microeng, 2019.

12. Engel, L., et al., Lattice micropatterning of electron microscopy grids for improved cellular cryoelectron tomography throughput. bioRxiv, 2020.

13. This research was supported in part by funds from the University of Wisconsin-Madison, Morgridge Institute for Research, and the National Institutes of Health (R01 GM104540, U24 GM139168, and R01 GM114561) to E.R.W. Cryo-EM data was collected at the University of Wisconsin-Madison, Department of Biochemistry Cryo-EM Research Center. 